\title{
Impact of polyunsaturated fatty acids on patient-important outcomes in children and adolescents with autism spectrum disorder: a systematic review
}

Franco De Crescenzo ${ }^{1,2,3^{*}}$ D. , Gian Loreto D’Alò ${ }^{1,4}$, Gian Paolo Morgano ${ }^{5}$, Silvia Minozzi ${ }^{1}$, Zuzana Mitrova ${ }^{1}$, Rosella Saulle ${ }^{1}$, Fabio Cruciani ${ }^{1}$, Francesca Fulceri ${ }^{6}$, Marina Davoli ${ }^{1}$, Maria Luisa Scattoni ${ }^{6}$, Francesco Nardocci ${ }^{6}$, Holger Jens Schünemann ${ }^{5,7,8}$, Laura Amato ${ }^{1}$ and on behalf of the ISACA guideline working group

\begin{abstract}
Background: Recent randomized controlled trials (RCTs) claimed PUFAs to be effective for autism spectrum disorder (ASD) but international guidelines have not considered yet this body of evidence. Our aim was to assess the effectiveness of PUFAs in children and adolescents with ASD, for the Italian national guidelines on the management of ASD in children and adolescents.

Methods: We performed a systematic review and meta-analysis of RCTs comparing PUFAs versus placebo or a healthy diet for the treatment of ASD in children and adolescents. The outcomes considered were deemed by the guideline panel to be highly relevant to children and adolescents with ASD and to their caregivers. The outcomes included hyperactivity, quality of sleep, self-harm, aggression, irritability, anxiety, attention, adaptive functioning, social interaction, restricted and repetitive interests and behavior, communication, hyperactivity and disruptive behaviors coexistent with core symptoms. The risk of bias of the included studies was assessed with the Cochrane tool, and the rating of the confidence in the effect estimates according to the Grading of Recommendations Assessment, Development and Evaluation (GRADE) approach.

Results: We included 9 studies with 405 participants. The strength of evidence ranged from low to very low. Six studies included preschoolers and school-age children, three studies included both children and adolescents. The majority of participants were males (83.8\%), with a mean age of 6.7 years. PUFAs were superior compared to placebo in reducing anxiety in individuals with ASD (SMD $-1.01,95 \% \mathrm{Cl}-1.86$ to -0.17 ; very low certainty of evidence). Moreover, PUFAs worsened quality of sleep compared to a healthy diet (SMD 1.11, 95\% Cl 0.21 to 2.00; very low certainty of evidence). PUFAs were not better than placebo in reducing aggression, hyperactivity, adaptive functioning, irritability, restricted and repetitive interests and behaviors and communication. Effects on some critical outcomes such as sleep, self-harm and disruptive behavior are currently unknown. The main limitations were the small number of participants included in the RCTs and the dosage which varied greatly (from $200 \mathrm{mg} /$ day to 1540 $\mathrm{mg} /$ day), making it difficult to address causal inference.

(Continued on next page)
\end{abstract}

\footnotetext{
* Correspondence: f.decrescenzo@deplazio.it

${ }^{1}$ Department of Epidemiology, Lazio Regional Health Service, Via Cristoforo

Colombo, 112, 00154 Rome, Italy

${ }^{2}$ Department of Psychiatry, University of Oxford, Oxford, UK

Full list of author information is available at the end of the article
}

(c) The Author(s). 2020 Open Access This article is distributed under the terms of the Creative Commons Attribution 4.0 International License (http://creativecommons.org/licenses/by/4.0/), which permits unrestricted use, distribution, and reproduction in any medium, provided you give appropriate credit to the original author(s) and the source, provide a link to the Creative Commons license, and indicate if changes were made. The Creative Commons Public Domain Dedication waiver (http://creativecommons.org/publicdomain/zero/1.0/) applies to the data made available in this article, unless otherwise stated. 
(Continued from previous page)

Conclusions: PUFAs did not show evidence of effect in children and adolescents with ASD and the certainty of evidence as measured with the GRADE was low to very low. Further research is needed on this topic because the available evidence is inconclusive.

Keywords: Autism spectrum disorder, Polyunsaturated fatty acids, Systematic review, Meta-analysis, Children, Adolescents

\section{Introduction}

Autism spectrum disorder (ASD) is characterized by abnormal neurodevelopment, with core symptoms consisting in persistent alterations in social interaction and communication, and restricted and repetitive interests and behaviors that cause reduced functioning, regardless of intellectual ability [1].

The prevalence of ASD in Italy is about 1.14-1.3\% [2, $3]$ ), and its prevalence in the world is between 1 and $2 \%$ [4]. A recent Italian study found a male: female ratio of about $4: 1$ [5], with $48 \%$ of children being affected by intellectual disability [5], data are consistent with the international literature [6].

Poly-unsaturated fatty acids (PUFAs) contain at least two carbon-carbon double bonds in their carboxylic chain, and can be classified according to the distance of the first double bond from the methyl group placed at the end of the molecule, into omega- 3 , omega- 6 and omega-9 (the latter is not essential in humans because they can be synthesized from carbohydrates or other fatty acids). Fish oils are rich in omega-3, plants are rich in omega- 6 , and two PUFAs, $\alpha$-linolenic acid (an omega3 fatty acid) and linoleic acid (an omega- 6 fatty acid) are essential nutrients in humans [7].

Eicosapentaenoic acid (EPA) and docosahexaenoic acid (DHA), are omega-3 fatty acids thought to favor a reduction in the synthesis of pro-inflammatory mediators. This effect has supported their use in the secondary prevention of hypertension, coronary artery disease, type 2 diabetes and in some other diseases [8], although their effect is controversial $[7,9,10]$. The role of EPA and DHA in disorders of the central nervous system has been extensively investigated in the last two decades [8]. The rationale behind the use of these agents in psychiatric disorders would be their primary action in producing modifications of the synaptic membrane, with implications in the transmission and transduction of the signal [8, 11]. Magnetic resonance imaging studies suggested that a reduced functional connection of longdistance brain areas is related to difficulties in social interactions in children and adolescents with autism spectrum disorder [12]. In mental health, EPA and DHA have been studied for the therapy of attention deficit hyperactivity disorder (ADHD), ASD, unipolar and bipolar affective disorders, anxiety disorder, obsessive- compulsive disorder, aggression, hostility, impulsivity, borderline personality disorder, substance use and anorexia nervosa $[8,13]$.

The risk of serious adverse events such as stroke, pulmonary embolism, and bleeding following PUFAs administration is still unclear, notwithstanding some recent RCTs showed a small increase of these events in the PUFAs arms [7, 14]. When fish oil is ingested for a long period of time (several months), it is better to ingest vitamin $\mathrm{E}$ (antioxidant) together, in preparation for lipid peroxidation. Also, as the toxicity of vitamin A or $\mathrm{D}$ could be increased, FDA recommends not ingesting more than $3 \mathrm{~g}$ of fish oil-derived omega-3 fatty acids per day [15]..

The goal of this systematic review was to assess the efficacy and safety of PUFAs in children and adolescents with ASD.

\section{Methods}

This systematic review was performed to support the development of the Italian National Institute of Health (ISS) guidelines for the diagnosis and management of children and adolescents with ASD. The ISS guideline group for the diagnosis and management of autism spectrum disorder, comprised of a multidisciplinary panel including caregivers of children/adolescents with ASD, formulated 15 questions for developing evidencebased health recommendations $[16,17]$ in accordance with the recently published ISS methodological manual for clinical practice guidelines (GL) development [18]. The Evidence Review Team together with the ISS principal investigator and the GL chairs decided to include two more questions for training the panel members on the pathway leading to the recommendations.

Using the GRADE approach, the panel began its work agreeing on a recommendation addressing the impact of PUFAs on patient-important outcomes in children and adolescents with autism spectrum disorder; a common question for this population.

\section{The questions}

Should PUFAs versus placebo be used for the treatment of children and adolescents with ASD?

Should PUFAs versus healthy diet be used for the treatment of children and adolescents with ASD? 


\section{Population}

Children and adolescents aged 0-18 years, of both genders, with a primary diagnosis of autism spectrum disorder. A concurrent secondary diagnosis of another health disturbance was not considered as an exclusion criterion.

\section{Intervention}

Any type and any dose of PUFAs, including eicosapentaenoic acid, docosahexaenoic acid, and $\alpha$-linolenic acid. We will include also studies in which fatty acids will be used as adjunctive treatment (for example, indicated in addition to behavioral or pharmacological interventions).

\section{Comparisons}

- Placebo or no intervention.

- Healthy diet.

\section{Outcomes}

The outcomes considered in this meta-analysis were deemed by the guideline panel to be highly relevant to children and adolescents with ASD. They were identified in accordance with the methods described in the ISS manual [18] and are the result of a group process conducted using the guideline development tool GRADEpro [19], which includes outcomes' generation and rating on a 9-point scale. Outcomes with a mean rating score from 6.33 to 9 were considered critical, from 3.33 to 6.32 important, from 1 to 3.32 not important for decision-making.

To measure the efficacy of the treatment, we assessed the following outcomes:

- Hyperactivity (critical),

- Quality of sleep (critical),

- Self-harm (critical),

- Aggression (critical),

- Irritability (critical),

- Anxiety (critical),

- Attention (critical),

- Adaptive functioning (critical),

- Social interaction (important),

- Restricted and repetitive interests and behavior (important),

- Communication (important),

- Hyperactivity and disruptive behaviors coexistent with core symptoms (important).

In addition, the evidence review team measured the tolerability of the treatment through the following outcomes:

- Discontinuation due to any cause (not important),

- Number of adverse events (not important).

\section{Types of studies included}

Randomized controlled trials comparing PUFAs with placebo or any other intervention in the treatment of autism spectrum disorder were included. Quasirandomized trials, such as those allocating by using alternate days of the week, and open-label trials were excluded. For trials that had a crossover design only results from the first randomization period were considered.

\section{Literature search}

A comprehensive computer literature search of the CENTRAL, PubMed/Medline, Embase, PsycINFO, Web Of Science databases was carried out up to October 2018. We also searched for ongoing clinical trials and unpublished trials. The full search strategy used is available in the supplementary materials, Additional file 1 . No date limit and no language restrictions were used.

\section{Study selection and data extraction}

Two reviewers (FDC, GD) independently screened titles and abstracts of all publications that were obtained by the search strategy. The same authors independently assessed the full text of potentially-relevant studies for inclusion. Disagreement was resolved by a consensus meeting or by a third reviewer (LA).

Two reviewers (FDC, GD) independently extracted data. We used a structured data abstraction form to ensure consistency of appraisal for each study. Information extracted included study characteristics (such as lead author, publication year, journal), participant characteristics (age range, setting, diagnosis), intervention details (such as dose ranges, mean doses of study drugs), length of follow up and outcome measures of interest.

\section{Data analysis}

Data were entered and analyzed using RevMan 5.3 software. Continuous outcomes were analyzed using standardized mean difference (SMD) with 95\% confidence intervals because different scales were used in the included studies. We combined data using the random effect model because a certain degree of heterogeneity was expected among trials [20]. In interpreting SMD values, we considered SMD "small" if $<0.40$, "moderate" from 0.40 to 0.70 , and "large" if $>0.7$. We analyzed dichotomous outcomes by calculating the risk ratio (RR) for each trial with the uncertainty in each result being expressed with $95 \%$ confidence interval (CI). Heterogeneity between studies has been investigated by the Q-test, by Isquared statistic (I-squared equal to or more than 50\% was considered indicative of heterogeneity), and by visual inspection of the forest plots. 


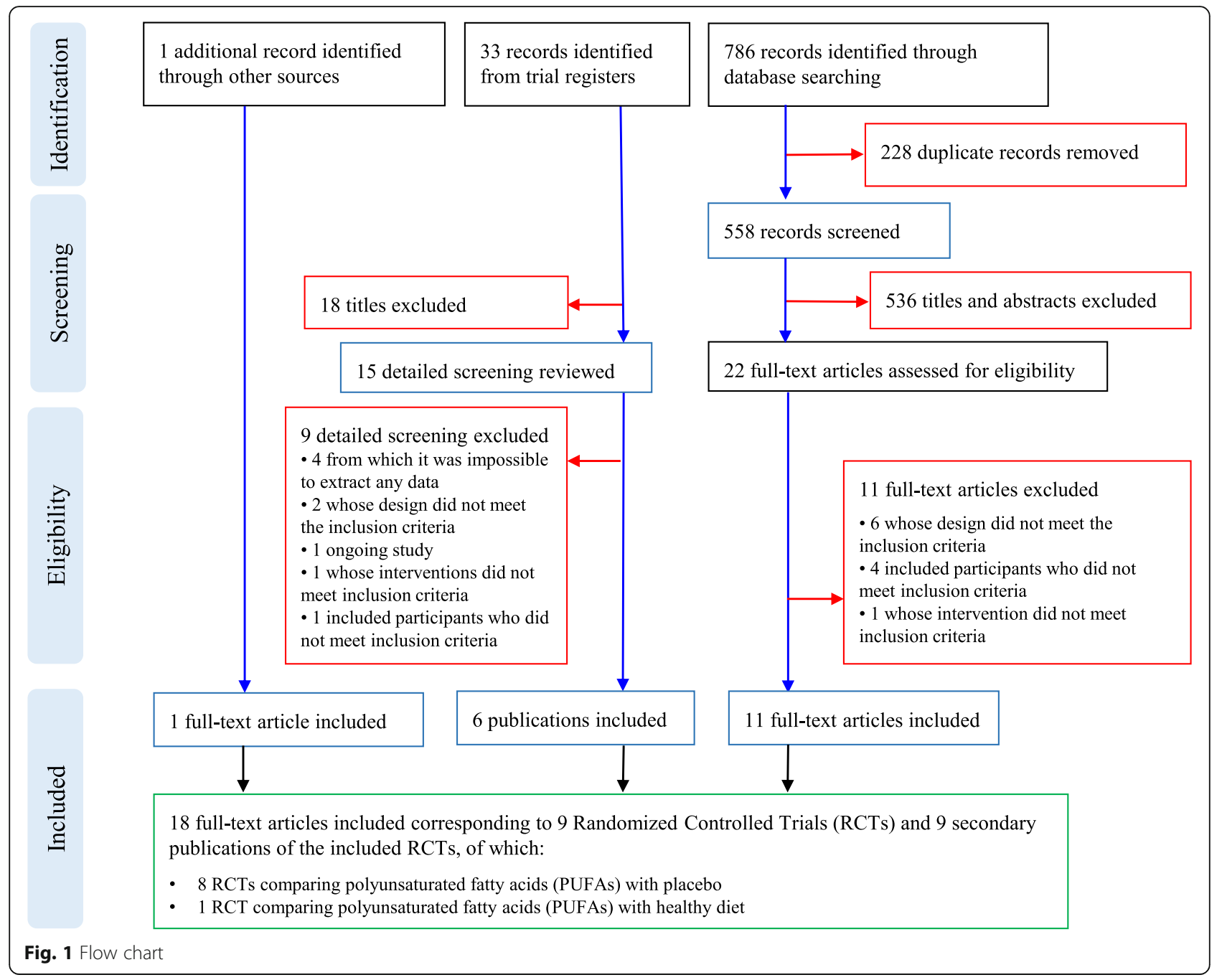

Risk of bias and overall certainty of evidence assessment Two authors independently (FDC, GD) assessed the risk of bias in the included studies using the tool described in the Cochrane Handbook for systematic reviews of interventions as a reference guide [21]. The following domains were assessed:

1. sequence generation;

2. allocation concealment;

3. blinding;

4. incomplete outcome data;

5. selective reporting;

6. other bias (e.g. funding source, baseline imbalance, interventions insufficiently well delivered).

A 'Risk of bias' table was created for the included studies, which indicates the study's performance in each of the above domains. For each domain, a judgment was assigned in terms of low risk of bias; high risk of bias; unclear risk of bias.
The main results of the review were presented in 'Summary of findings' (SoF) tables, as recommended by Cochrane [22]. We produced the SoF tables for estimates based on the methodology developed from the Grading of Recommendations Assessment, Development and Evaluation (GRADE) Working Group [23]. For more details, see $[24,25]$. We rated the confidence in the effect estimates considering study limitations, indirectness, inconsistency, imprecision of effect estimates, and risk of publication bias. According to the software GRADEpro GDT 2014, four levels of certainty in the evidence were assigned: high, moderate, low, very low.

Three authors (FDC, SV, RS) applied the GRADE approach to evaluating the certainty of evidence for the outcomes considered as "critical", "important", or "not important" from the members of the panel:

- Efficacy (hyperactivity, quality of sleep, self-harm, aggression, irritability, anxiety, attention, adaptive functioning, social interaction, restricted and repetitive interests and behavior, communication, hyperactivity 
and disruptive behaviors coexistent with core symptoms);

- Tolerability (discontinuation due to any cause, number of adverse events).

\section{Results}

\section{Selected studies}

From databases searches, we retrieved 786 citations of which 228 were removed, being duplicates. Of the 558 remaining documents, 22 studies were evaluated in full text as potentially relevant. Of these, eleven were excluded. Among the excluded, six studies were not RCTs [26-31], four studies included preterm infants between 18 and 36 months with a high risk of ASD [32-35], one study assessed an intervention that did not meet inclusion criteria [36]. We retrieved further 33 records from trial registers, 15 of which were evaluated in full text. We found four completed clinical trials of which we were unable to obtain any result [37-40]; two trials whose design did not meet inclusion criteria [41, 42]; one ongoing study [43]; one trial whose intervention did not meet inclusion criteria [44]; and one trial whose participants did not meet inclusion criteria [45]. We also retrieved one full-text document from other sources [46] (Fig. 1).

Finally, a total of 9 studies with 405 participants (18 documents), were included (see Additional file 7: references for included and excluded trials).

\section{Study characteristics}

Six studies $(66.7 \%)$ included preschoolers and school-age children, while three studies (33.3\%) included both children and adolescents. The majority of participants were males (83.8\%), with a mean age of 6.7 years. In seven studies the diagnosis was performed using the DSM-IV criteria, while one study used the DSM-5 criteria [46] and another study based the diagnosis on parent reports [47]. Seven studies reported the use of scales for support in the diagnosis, such as the Autism Diagnostic InterviewRevised (ADI-R), Autism Diagnostic Observation Scale (ADOS), Childhood Autism Rating Scale (CARS), Social Communication Questionnaire (SCQ). All individuals included in the selected studies were outpatients.

Eight studies compared PUFAs vs placebo [12, 46-52], and one vs healthy diet [53].

We considered healthy diet as something closed to no intervention because is part of standard clinical care.

Overall, 201 participants were randomly assigned to PUFAs, 161 to placebo, 13 to a healthy diet, and 30 to other interventions (i.e. Vitamin D supplementation). The mean study sample size was 45 participants, ranging between 13 [48] and 110 [46] participants. Two studies recruited patients from Europe, five from North America, one from Asia and one from Oceania. Study median duration was twelve weeks (range: 6-52).
The scales used to measure the outcomes of interest included the Aberrant Behavior Checklist (ABC), the Behavior Assessment System for Children (BASC), the Clinical Global Impression-Improvement scale (CGI-I), the Clinical Global Impression-Severity scale (CGI-S), the Expressive Vocabulary Test (EVT), the Mullen Scales of Early Learning, the Preschool Language Scale (PLS), the Peabody Picture Vocabulary Test (PPVT), the Social Responsiveness Scale (SRS), the Vineland Adaptive Behavior Scale (VABS).

Regarding "hyperactivity" outcome, data were extracted from ABC - hyperactivity subscale [9, 42-45]; for the outcome "quality of sleep", we used the CBCL sleep subscale [51]; for "aggression", we selected BASC externalizing subscale [49] and CBCL - aggression subscale [53]; for the outcome "irritability", we selected $\mathrm{ABC}$ - irritability subscale [9, 12, 46-48]; for the outcome "anxiety", we extracted data regarding the BASC internalizing subscale [49] and CBCL - anxious/ dependent subscale [53]; for the outcome "attention", we selected CBCL - attention subscale [53]; for "adaptive functioning", we used the BASC - adaptive skill subscale [49] and the item social skills - parents assessed of the subscale adaptive skills of the BASC [52]; for the outcome "social interaction", we selected the SRS total score $[12,47,49,51]$, and, when this scale was not assessed, the $A B C$ - social withdrawal subscale $[46,48]$, or the BASC - withdrawn subscale [53]; for the outcome "restricted and repetitive interests and behaviors" we selected the $A B C$ - stereotypy subscale [46-49], and from SRS - autistic mannerisms subscale [51]; for "communication" outcome, we selected data from ABC - inappropriate speech subscale [46-48], from EVT [49], from SRS - social communication subscale [12, 51], and from MSEL - expressive language subscale [53].

As regards to the composition of PUFAs, in five trials a combination of EPA and DHA were administered, while in four studies only DHA was prescribed. Doses of EPA ranged from $693 \mathrm{mg}$ [51] to $840 \mathrm{mg} /$ day [48], while doses of DHA ranged from $200 \mathrm{mg}$ [52] to $722 \mathrm{mg} /$ day [46].

Overall PUFAs dosage varied greatly in the studies analyzed, with a median dose of $1155 \mathrm{mg} /$ day, ranging from a minimum of $200 \mathrm{mg} /$ day [52] to a maximum of $1540 \mathrm{mg} /$ day [48]. Dropout rates varied between $7.7 \%$ [48] and 29.6\% [49]; in two studies [12, 53] dropouts were not clearly reported. We reported full clinical and demographic characteristics and description of interventions in Table 1.

\section{Data analysis and overall certainty of evidence assessment}

We presented the forest plots for specific outcomes for the comparisons PUFAs versus placebo in Additional file 3 and PUFAs versus healthy diet in Additional file 4 . 


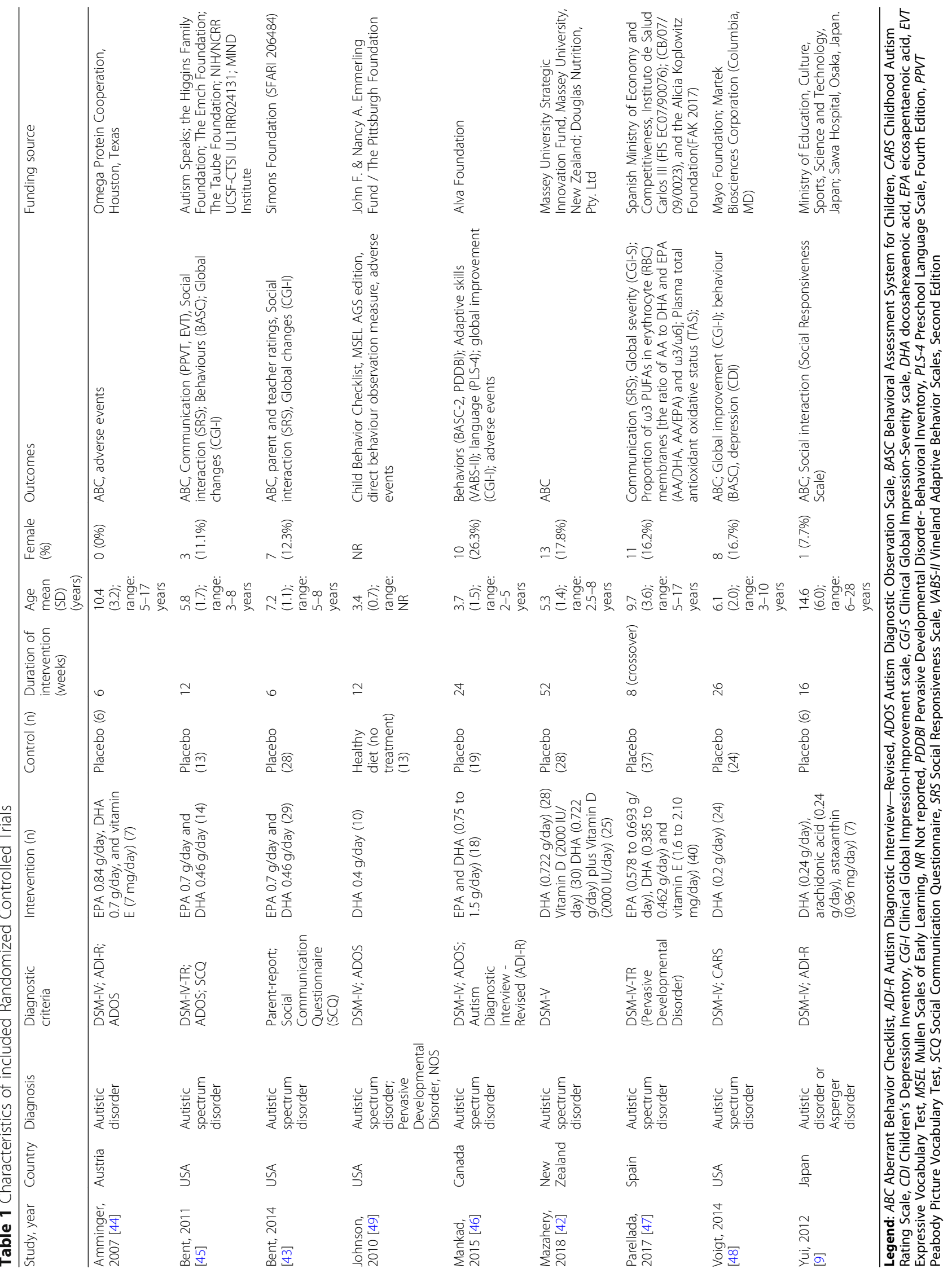


Heterogeneity $\left(\mathrm{I}^{2}\right)$ across considered outcomes was between 0 and $58 \%$ in the comparison of PUFAs versus placebo, while there was no heterogeneity in the comparison between PUFAs and healthy diet since only one trial was included (Additional file 3 and Additional file 4). Our judgment on inconsistency is shown in Additional file 5 and Additional file 6.

\section{Discussion}

We conducted a systematic review and meta-analysis on efficacy and tolerability of the use of PUFAs compared to placebo or a healthy diet for children and adolescents with ASD. We found that despite some increase in number and quality of studies on PUFAs for children and adolescents with ASD over time (six RCTs were published in the last five years), results remained preliminary. PUFAs did not show evidence of effect for children and adolescents with ASD and the certainty of evidence as measured with the GRADE was low to very low.

This systematic review and meta-analysis is based on 9 studies, including 201 children and adolescents randomly assigned to PUFAs, 161 to placebo and 13 to healthy diet. Our systematic search was comprehensive and to our knowledge, this is the most up-to-date synthesis of data on this field.

This study has some limitations. First, the comparison healthy diet had a small number of studies included (only one RCT) and a very small number of participants (13 children and adolescents with ASD), limiting the evidence and the generalizability of the results.

Second, some of the outcomes which were considered as critical or important were not assessed by any study (i.e. for the comparison PUFAs versus placebo: quality of sleep, self-harm, attention, hyperactivity and disruptive behaviors coexistent with core symptoms; for PUFAs versus healthy diet: hyperactivity, self-harm, irritability, adaptive functioning, restricted and repetitive interests and behaviors, hyperactivity and disruptive behaviors coexistent with core symptoms).

Third, dosage varied greatly, from a minimum of 200 $\mathrm{mg} /$ day [52] to a maximum of $1540 \mathrm{mg} /$ day [44], making it difficult to address causal inference. International agencies as well do not fully agree on the dietary recommended intake for PUFAs. As for omega-3 fatty acids for infants, the WHO suggests $400 \mathrm{mg}$ per $10 \mathrm{~kg}$ body weight $[15,55]$, while the International Scientific Society of Fatty Acids and Lipids (ISSFAL) suggests $350-750 \mathrm{mg}$ per $10 \mathrm{~kg}$ body weight [56]. Regarding the maximum tolerable dose of omega-3, the Food and Drug Administration (FDA) recommends not to take more than $3 \mathrm{~g} /$ day of EPA and DHA, of which up to 2d/day through supplements [57]. The daily limitation aims to limit the intake of fat-soluble vitamins, such as Vitamin A and Vitamin D [15, 58]. The Institute of Medicine (IOM) has not established a tolerable Upper Intake Level (UL) for omega-3 intake, but has shown that high doses (more than $900 \mathrm{mg} /$ day of EPA plus $600 \mathrm{mg} /$ day of DHA) may reduce the immune response, while doses between 2 and $15 \mathrm{~g}$ of EPA and/or DHA may have negative effects on coagulation, promoting bleeding [59]. According to the European Food Safety Authority (EFSA), however, supplementation with doses up to $5 \mathrm{~g} /$ day of EPA and/or DHA would be safe, as no side effects have been found regarding bleeding and immune response [60].

Fourth, our reviews did not take into account the difference between nutraceuticals and pharmacological products. Indeed, a recent systematic review highlighted differences in safety between nutraceuticals and pharmacological PUFAs, pointing out that prescribed pharmacological products are supported by robust clinical development and safety monitoring programs, while nutraceuticals are not required to demonstrate safety or efficacy before marketing [61]. Nutraceuticals may also contain potentially harmful components, including other lipids, cholesterol and toxins, and are not produced in Good Manufacturing Practice (GMP), while pharmacological products contain high purity DHA and/or EPA $[61,62]$.

Fifth, we did not prospectively register the protocol for our systematic review, and this is a study limitation. However, the clinical question was formulated by a multidisciplinary panel of experts, and the methodology followed for the development of the systematic review was based on the manual developed and published by the ISS $[18,63]$.

Previous systematic reviews on PUFAs for children and adolescents with ASD included respectively two RCTs [64], four RCTs [65], and five RCTs [66] concluding that there was no evidence of effect $[64,66]$ or that PUFAs could potentially improve some ASD symptoms [65]. The differences between our findings and the one of the meta-analysis of RCTs by Mazahery et al. [65], especially when considering the efficacy of PUFAs on communication outcome they found (4 RCTs; MD -1.96, $95 \%$ CI -3.57 to -0.34 ), could be partially due to the different methods used: the authors performed their analyses extracting the mean change and SD of change from baseline to endpoint. Moreover, we used the Standardized Mean Difference to pool data from assessed through different scales, while Mazahery et al. extracted only data regarding $A B C$ subscales. Horvath et al. [66] performed different meta-analyses for each instrument used to assessed the outcomes of interest; they found PUFAs to be efficacious in improving lethargy-social withdrawal (2 RCTs; MD 1.98, 95\% CI 0.32 to 3.63) when assessed with the $\mathrm{ABC}$, and daily-living (1 RCT; MD 6.2, 95\% CI 0.37 to 12.03) as assessed by VABS. Also, the authors found the PUFAs to worsen 
externalizing behavior (2 RCTs; MD -6.22, 95\% CI 10.9 to -1.59 ) and social skills (1 RCT; MD -7.0, 95\% CI -13.62 to -0.38 ) as assessed through BASC. The most recent RCT published [46], not included in the previous systematic reviews, suggested that PUFAs could improve some core symptoms of ASD, but its findings, when pooled with other RCTs results, did not translate into statistical significance for any outcome in our metaanalysis. These mixed findings are in line with the very low and low certainty of evidence found in our study by using the GRADE. Notwithstanding the publication of numerous RCTs in recent years comparing PUFAs against placebo, their sample size was always small. The consequence was that the most frequent reason for lowering the certainty of evidence in our systematic review was the insufficient sample size. It therefore appears necessary to conduct larger RCTs to establish the efficacy of PUFA in this population; this would be even more true if the size of the effect to be highlighted were small.

Although the efficacy of PUFAs in children and adolescents with ASD is still controversial, PUFAs are administered to a portion of this population ranging from 18 to $51 \%[67,68]$, probably thanks to the fact that costs and difficulties of implementation appear negligible [69], and safety concerns seem small [65]. Also the rationale behind the administration of PUFAs in individuals with ASD, i.e. that the observation of reduced plasma concentrations of EPA and DHA in meta-analyses of casecontrol studies would be due to inefficient or disrupted metabolism, could instead be due to the action of confounding factors, i.e. selective diets [65], and needs to be demonstrated through good quality studies, controlled for possible confounders.

\section{Conclusions}

In conclusion, we found no evidence of efficacy for PUFAs versus placebo on hyperactivity, aggression, irritability, adaptive functioning, social interaction, restricted and repetitive interests and behaviours, communication, with very low to low certainty of evidence, evidence of efficacy on anxiety only, with very low certainty of evidence and evidence of a negative effect on quality of sleep, with very low certainty of evidence. We found no evidence of efficacy of PUFAs versus healthy diet on aggression, anxiety, attention, social interaction, communication with very low certainty of evidence and we found efficacy on quality of sleep, with very low certainty of evidence. No clinical recommendation can be suggested at the present time. We do not believe the evidence is strong enough to allow the construction of a phase III trial, while phase II, dose-findings trials are necessary to ascertain the dose and the effect of PUFAs for children and adolescents with ASD.

\section{Supplementary information}

Supplementary information accompanies this paper at https://doi.org/10. 1186/s12955-020-01284-5.

Additional file 1. Search strategy and results

Additional file 2. Risk of Bias Summary

Additional file 3. Forest plots of comparisons between PUFAs and Placebo

Additional file 4. Forest plots of comparisons between PUFAs and healthy diet

Additional file 5. Evidence profile - PUFAs versus Placebo

Additional file 6. Evidence profile - PUFAs versus healthy diet

Additional file 7. References for included and excluded trials

Additional file 8. PRISMA checklist

\section{Abbreviations}

ABC: Aberrant Behavior Checklist; ADHD: attention deficit hyperactivity disorder; ASD: autism spectrum disorder; BASC: Behavior Assessment System for Children; CGI-I: Clinical Global Impression-Improvement scale; CGIS: Clinical Global Impression-Severity scale; Cl: Confidence interval;

DHA: Docosahexaenoic acid; EPA: Eicosapentaenoic acid; EVT: Expressive Vocabulary Test; GRADE: Grading of Recommendations Assessment, Development and Evaluation; MSEL: Mullen Scales of Early Learning; PLS: Preschool Language Scale; PPVT: Peabody Picture Vocabulary Test; PUFA: polyunsaturated fatty acid; RCT: randomized controlled trial; RR: risk ratio; SMD: standardized mean difference; SRS: Social Responsiveness Scale; VABS: Vineland Adaptive Behavior Scale

\section{Acknowledgements}

Collaborating author names from the ISACA guideline working group: Raffaella, Tancredi; Angelo, Massagli; Giovanni, Valeri; Corrado, Cappa; Serafino, Buono; Giuseppe, M, Arduino; Alessandro, Zuddas; Laura, Reali; Massimo, Molteni; Claudia, Felici; Concetta, Cordò; Lorella, Venturini; Cristina, Bellosio; Emanuela, Di Tommaso; Sandra, Biasci; Clelia, M, Duff.

\section{Author's contributions}

The author(s) read and approved the final manuscript.

\section{Funding}

No financial support was used for this research. Conceptualization: FDC, GLD, GPM, SM, HJS, LA.

Data curation: FDC, GLD, SM, ZM, RS, FC, MD, LA. Formal analysis: FDC, GLD, SM, LA.

Project administration: MD, MLS, FN, HJS, LA

Supervision: SM, MD, MLS, FN, HJS, LA.

Writing - original draft: FDC, GLD, GPM.

Writing - review \& editing: SM, ZM, RS, FC, FF, MD, MLS, FN, HJS, LA.

\section{Availability of data and materials}

All data supporting our findings is contained within the manuscript and the additional files.

The authors and can be contacted at f.decrescenzo@deplazio.it. (FDC) for further clarification, if required.

Ethics approval and consent to participate Not applicable.

Consent for publication

Not applicable.

\section{Competing interests}

The authors declare that they have no competing interests.

\section{Author details}

'Department of Epidemiology, Lazio Regional Health Service, Via Cristoforo Colombo, 112, 00154 Rome, Italy. ${ }^{2}$ Department of Psychiatry, University of Oxford, Oxford, UK. ${ }^{3}$ Pediatric University Hospital-Department (DPUO), Bambino Gesù Children's Hospital, Rome, Italy. ${ }^{4}$ School of Hygiene and 
Preventive Medicine, University of Rome Tor Vergata, Rome, Italy. ${ }^{5}$ Department of Health Research Methods, Evidence and Impact (formerly Clinical Epidemiology and Biostatistics), McMaster GRADE Centre, McMaster University, Hamilton, Canada. ${ }^{6}$ Research Coordination and Support Service, Istituto Superiore di Sanità, Viale Regina Elena 299, 00161 Rome, Italy. ${ }^{7}$ Michael G DeGroote Cochrane Canada Centre, McMaster University, Hamilton, Canada. ${ }^{8}$ Department of Medicine, McMaster University, Hamilton, Canada.

\section{Received: 28 March 2019 Accepted: 7 February 2020}

\section{Published online: 17 February 2020}

\section{References}

1. Lord C, Elsabbagh M, Baird G, Veenstra-Vanderweele J. Autism spectrum disorder. Lancet. 2018;392(10146):508-20.

2. Narzisi A, Posada M, Barbieri F, Chericoni N, Ciuffolini D, Pinzino M, et al. Prevalence of autism Spectrum disorder in a large Italian catchment area: a school-based population study within the ASDEU project. Epidemiol Psychiatr Sci. 2018;29:e5.

3. Istituto Superiore di Sanità. Giornata mondiale della Consapevolezza dell'Autismo: in Italia un bimbo ogni 77. 2019. Available at: https://www.iss. it/?p=3421. Accessed: 31 Dec 2019 .

4. CDC. Summary of Autism Spectrum Disorder (ASD) Prevalence Studies. Available at: www.cdc.gov/ncbddd/autism/documents/ ASDPrevalenceDataTable2016.pdf. Accessed 31 Dec 2018

5. Postorino V, Fatta LM, Sanges V, Giovagnoli G, De Peppo L, Vicari S, et al. Intellectual disability in autism Spectrum disorder: investigation of prevalence in an Italian sample of children and adolescents. Res Dev Disabil. 2016:48:193-201.

6. Lai MC, Lombardo MV, Baron-Cohen S. Autism Lancet. 2014;383(9920):896-910.

7. Abdelhamid AS, Martin N, Bridges C, Brainard JS, Wang X, Brown TJ, et al. Polyunsaturated fatty acids for the primary and secondary prevention of cardiovascular disease. Cochrane Database Syst Rev. 2018;7:CD012345.

8. Bozzatello P, Brignolo E, De Grandi E, Bellino S. Supplementation with Omega-3 Fatty Acids in Psychiatric Disorders: A Review of Literature Data. J Clin Med. 2016;5(8): 67

9. Manson JE, Cook NR, Lee IM, Christen W, Bassuk SS, Mora S, et al; VITAL Research Group. Marine n-3 fatty acids and prevention of cardiovascular disease and cancer. N Engl J Med 2019;380(1):23-32.

10. ASCEND Study Collaborative Group, Bowman L, Mafham M, Wallendszus K, Stevens W, Buck G, Barton J, Murphy K, et al. Effects of n-3 fatty acid supplements in diabetes mellitus. N Engl J Med. 2018;379(16):1540-50.

11. Foti F, De Crescenzo F, Vivanti G, Menghini D, Vicari S. Implicit learning in individuals with autism spectrum disorders: a meta-analysis. Psychol Med. 2015:45(5):897-910.

12. Yui K, Koshiba M, Nakamura S, Kobayashi Y. Effects of large doses of arachidonic acid added to docosahexaenoic acid on social impairment in individuals with autism spectrum disorders: a double-blind, placebocontrolled, randomized trial. J Clin Psychopharmacol. 2012;32(2):200-6.

13. De Giorgi R, De Crescenzo F, D'Alò GL, Rizzo Pesci N, Di Franco V, Sandini C, et al. Prevalence of Non-Affective Psychoses in Individuals with Autism Spectrum Disorders: A Systematic Review. J Clin Med. 2019;8(9).

14. Siscovick DS, Barringer TA, Fretts AM, Wu JH, Lichtenstein AH, Costello RB, et al. American Heart Association Nutrition Committee of the Council on Lifestyle and Cardiometabolic Health; Council on Epidemiology and Prevention; Council on Cardiovascular Disease in the Young; Council on Cardiovascular and Stroke Nursing; and Council on Clinical Cardiology. Omega-3 Polyunsaturated Fatty Acid (Fish Oil) Supplementation and the Prevention of Clinical Cardiovascular Disease: A Science Advisory From the American Heart Association. Circulation. 2017;135(15):e867-84.

15. Lee J-H. Polyunsaturated fatty acids in children. Pediatr Gastroenterol Hepatol Nutr. 2013;16(3):153-61.

16. IOM (Institute of Medicine). Clinical Practice Guidelines We Can Trust. Washington, DC: The National Academies Press, 2011.

17. Qaseem A, Forland F, Macbeth F, Ollenschläger G, Phillips S, Van der Wees $P$. Board of Trustees of the guidelines international network. Guidelines international network: toward international standards for clinical practice guidelines. Ann Intern Med. 2012 Apr 3;156(7):525-31.

18. Istituto Superiore di Sanità - Centro Nazionale per l'Eccellenza Clinica, la Qualità e la Sicurezza delle Cure. Manuale metodologico per la produzione di linee guida di pratica clinica. 2018. Available at: https://snlg.iss.it/wpcontent/uploads/2018/10/MM_v1.2 lug-2018.pdf Accessed 17 Gen 2019.

19. GRADEpro Guideline Development Tool [Software]. McMaster University, 2015 (developed by Evidence Prime, Inc.). Available from gradepro.org.

20. DerSimonian R, Laird N. Meta-analysis in clinical trials. Control Clin Trials. 1986;7(3):177-88.

21. Higgins JPT, Green S (editors). Cochrane Handbook for Systematic Reviews of Interventions Version 5.1.0 [updated March 2011]. The Cochrane Collaboration, 2011. Available from www.handbook.cochrane.org.

22. Schünemann HJ, Oxman AD, Higgins JPT, Vist GE, Glasziou P, Guyatt GH, et al. Presenting results and "Summary of findings" tables. In: Higgins JPT, Green S, eds. Cochrane handbook for systematic reviews of interventions. Chichester: Wiley; 2008. p. 335-8.

23. Guyatt GH, Oxman AD, Vist GE, Kunz R, Falck-Ytter Y, Alonso-Coello P, et al. GRADE: an emerging consensus on rating quality of evidence and strength of recommendations. BMJ. 2008;336:924-6.

24. Guyatt $G H$, Oxman AD, Santesso N, Helfand M, Vist G, Kunz R, et al. GRADE guidelines: 12. Preparing summary of findings tables-binary outcomes. J Clin Epidemiol. 2013;66(2):158-72

25. Guyatt GH, Thorlund K, Oxman AD, Walter SD, Patrick D, Furukawa TA, et al. GRADE guidelines: 13. Preparing summary of findings tables and evidence profiles-continuous outcomes. J Clin Epidemiol. 2013;66(2):173-83.

26. Johnson SM, Hollander E. Evidence that eicosapentaenoic acid is effective in treating autism. J Clin Psychiatry. 2003;64:848-9.

27. Meguid NA, Atta HM, Gouda AS, Khalil RO. Role of polyunsaturated fatty acids in the management of Egyptian children with autism. Clin Biochem. 2008:41:1044-8.

28. Meiri G, Bichovsky Y, Belmaker RH. Omega 3 fatty acid treatment in autism. J Child Adolesc Psychopharm. 2009:19(4):449-51.

29. Patrick $L$, Salik R. The effect of essential fatty acid supplementation on language development and learning skills in autism and Asperger's syndrome. Autism Asperger's Digest. 2005;Jan-Feb:36-7.

30. Politi P, Cena H, Comelli M, Marrone G, Allegri C, Emanuele E, et al. Behavioral effects of omega-3 fatty acid supplementation in young adults with severe autism: an open label study. Arch Med Res. 2008; 39(7):682-5.

31. Ooi YP, Weng SJ, Jang LY, Low L, Seah J, Teo S, et al. Omega-3 fatty acids in the management of autism spectrum disorders: findings from an open-label pilot study in Singapore. Eur J Clin Nutr. 2015;69(8):969-71.

32. Boone KM, Gracious B, Klebanoff MA, Rogers LK, Rausch J, Coury DL, et al. Omega-3 and -6 fatty acid supplementation and sensory processing in toddlers with ASD symptomology born preterm: a randomized controlled trial. Early HumDev. 2017;115:64-70.

33. Boone KM, Gracious B, Klebanoff MA, Rogers LK, Rausch J, Coury DL, et al. Corrigendum to "Omega-3 and -6 fatty acid supplementation and sensory processing in toddlers with ASD symptomology born preterm: a randomized controlled trial". Early Hum Dev. 2018;128:120.

34. Keim SA, Gracious B, Boone KM, Klebanoff MA, Rogers LK, Rausch J, et al. $\omega$ 3 and $\omega-6$ fatty acid supplementation may reduce autism symptoms based on parent report in preterm toddlers. J Nutr. 2018;148(2):227-35.

35. Sheppard KW, Boone KM, Gracious B, Klebanoff MA, Rogers LK, Rausch J, et al. Effect of Omega-3 and -6 supplementation on language in preterm toddlers exhibiting autism Spectrum disorder symptoms. J Autism Dev Disord. 2017:47(11):3358-69.

36. Adams JB, Audhya T, Geis E, Gehn E, Fimbres V, Pollard EL, et al. Comprehensive nutritional and dietary intervention for autism Spectrum disorder-a randomized, controlled 12-month trial. Nutrients. 2018;10(3):E369.

37. ClinicalTrials.gov. Bethesda (MD): National Library of Medicine (US). Identifier NCT01248130. Omega-3 Fatty Acids Monotherapy in Children and Adolescents With Autism Spectrum Disorders. (first posted $25^{\text {th }}$ November 2010).

38. ClinicalTrials.gov. Bethesda (MD): National Library of Medicine (US). Identifier NCT01260961. Developing Treatment, Treatment Validation and Treatment Scope in the Setting of an Autism Clinical Trial. (first posted 15th December 2010).

39. ClinicalTrials.gov. Bethesda (MD): National Library of Medicine (US). Identifier NCT00467818. Omega 3 Fatty Acids in the Treatment of Children With Autism Spectrum Disorders. (first posted 1st May 2007).

40. ClinicalTrials.gov. Bethesda (MD): National Library of Medicine (US). Identifier NCT03620097. Evaluate the Efficacy and Safety of DHA in the Adjuvant Treatment of Children With ASD (first posted $8^{\text {th }}$ August 2018). 
41. ClinicalTrials.gov. Bethesda (MD): National Library of Medicine (US). Identifier NCT03757585. Management of Emotional Dysregulation in Youth With Nonverbal Learning Disability (NVLD) and/or Autism Spectrum Disorders (ASD) Using Telepsychiatry of Complementary and Alternative Treatments. clinicaltrial.gov (first posted 29th November 2018).

42. ClinicalTrials.gov. Bethesda (MD): National Library of Medicine (US). Identifier NCT01695200. Omega-3 Fatty Acids in Autism Spectrum Disorders. clinicaltrial.gov (first posted 27th September 2012).

43. ClinicalTrials.gov. Bethesda (MD): National Library of Medicine (US). Identifier NCT03550209. Fatty Acid Supplementation in Children With ASD (Omega Heroes). clinicaltrial.gov (first posted 8th June 8 2018).

44. ClinicalTrials.gov. Bethesda (MD): National Library of Medicine (US). Identifier NCT02059577. Nutritional and Dietary Treatment Study for Children/Adults With Autism. clinicaltrial.gov (first posted $11^{\text {th }}$ Febraury 2014).

45. ClinicalTrials.gov. Bethesda (MD): National Library of Medicine (US). Identifier NCT01683565. Preemie Tots: A Pilot Study to Understand the Effects of Prematurity in Toddlerhood. clinicaltrial.gov (first posted: $12^{\text {th }}$ September 2012)

46. Mazahery $\mathrm{H}$, Conlon CA, Beck KL, Mugridge O, Kruger MC, Stonehouse W, et al. A randomised controlled trial of vitamin D and omega-3 long chain polyunsaturated fatty acids in the treatment of irritability and hyperactivity among children with autism spectrum disorder. J Steroid Biochem Mol Biol. 2019:187:9-16.

47. Bent S, Hendren RL, Zandi T, Law K, Choi JE, Widjaja F, et al. Internet-based, randomized, controlled trial of omega-3 fatty acids for hyperactivity in autism. J Am Acad Child Adolesc Psychiatry. 2014;53(6):658-66.

48. Amminger GP, Berger GE, Schafer MR, Klier C, Friedrich MH, Feucht M. Omega3 fatty acids supplementation in children with autism: a double-blind randomized, placebo-controlled pilot study. Biol Psychiatry. 2007;61:551-3.

49. Bent S, Bertoglio K, Ashwood P, Bostrom A, Hendren RL. A pilot randomized controlled trial of omega-3 fatty acids for autism spectrum disorder. J Autism Dev Disord. 2011:41(5):545-54

50. Mankad D, Dupuis A, Smile S, Roberts W, Brian J, Lui T, et al. A randomized, placebo controlled trial ofomega-3 fatty acids in the treatment of young children with autism. Mol Autism. 2015;6:18.

51. Parellada M, Llorente C, Calvo R, Gutierrez S, Lázaro L, Graell M, et al. Randomized trial of omega-3 for autism spectrum disorders: effect on cell membrane composition and behavior. Eur Neuropsychopharmacol. 2017; 27(12):1319-30.

52. Voigt RG, Mellon MW, Katusic SK, Weaver AL, Matern D, Mellon B, et al. Dietary docosahexaenoic acid supplementation in children with autism. J Pediatr Gastroenterol Nutr. 2014;58(6):715-22.

53. Johnson CR, Handen BL, Zimmer M, Sacco K. Polyunsaturated fatty acid supplementation in young children with autism. J Dev Phys Disabil. 2010;22: $1-10$.

54. Sterne JAC, Egger M, Moher D; Cochrane Bias Method Group. Addressing reporting biases. In: Higgins JPT, Green S, editors. Cochrane Handbook of Systematic Reviews of Interventions. Chichester, UK: Wiley, 2008. p. 297-333.

55. WHO/FAO Expert Consultation on Diet, Nutrition and the Prevention of Chronic Diseases (2002: Geneva, Switzerland) Diet, nutrition and the prevention of chronic diseases: Report of a joint WHO/FAO expert consultation, Geneva, 28 January -- 1 February 2002. WHO technical report series 916.

56. International Society for the Study of Fatty Acids and Lipids (June 2004). Report of the Sub-Committee on Recommendations for Intake of Polyunsaturated Fatty Acids in Healthy Adults. [online]. Available at: http:// www.issfal.org/news-links/resources/publications/

PUFAlntakeReccomdFinalReport.pdf, undefined).

57. National Institutes of Health - Office of Dietary Supplements. Omega-3 fatty acids - Fact Sheet for Health Professionals. Disponibile su: https://ods.od.nih. gov/factsheets/Omega3FattyAcids-HealthProfessional/ Ultimo accesso: 2611-2018.

58. Bays HE. Safety considerations with omega-3 fatty acid therapy. Am J Cardiol. 2007:99(6A):35C-43C.

59. Institute of Medicine, Food and Nutrition Board. Dietary reference intakes for energy, carbohydrate, fiber, fat, fatty acids, cholesterol, protein, and amino acids (macronutrients). Washington, DC: National Academy Press; 2005.

60. EFSA Panel on Dietetic Products NaA. Scientific opinion on the tolerable upper intake level of eicosapentaenoic acid (EPA), docosahexaenoic acid (DHA) and docosapentaenoic acid (DPA). EFSA J. 2012;10:2815.
61. Hilleman D, Smer A. Prescription Omega-3 fatty acid products and dietary supplements are not interchangeable. Manag Care. 2016;25(1):46-52.

62. Santini A, Cammarata SM, Capone G, lanaro A, Tenore GC, Pani L, et al. Nutraceuticals: opening the debate for a regulatory framework. Br J Clin Pharmacol. 2018;84(4):659-72.

63. Morgano GP, Fulceri F, Nardocci F, Barbui C, Ostuzzi G, Papola D, et al. Introduction and methods of the evidence-based guidelines for the diagnosis and management of autism Spectrum disorder by the Italian National Institute of health. Submitted to Health Qual Life Outcomes.

64. James S, Montgomery P, Williams K. Omega-3 fatty acids supplementation for autism spectrum disorders (ASD). Cochrane Database Syst Rev. 2011;11: CD007992.

65. Mazahery H, Stonehouse W, Delshad M, Kruger MC, Conlon CA, Beck KL, et al. Relationship between Long Chain n-3 Polyunsaturated Fatty Acids and Autism Spectrum Disorder: Systematic Review and Meta-Analysis of CaseControl and Randomised Controlled Trials. Nutrients. 2017;9(2). pii: E155.

66. Horvath A, Łukasik J, Szajewska H. $\omega$-3 fatty acid supplementation does not affect autism Spectrum disorder in children: a systematic review and metaanalysis. J Nutr. 2017;147(3):367-76.

67. Hopf KP, Madren E, Santianni KA. Use and perceived effectiveness of complementary and alternative medicine to treat and manage the symptoms of autism in children: a survey of parents in a community population. J Altern Complement Med. 2016;22(1):25-32.

68. Huang A, Seshadri K, Matthews TA, Ostfeld BM. Parental perspectives on use, benefits, and physician knowledge of complementary and alternative medicine in children with autistic disorder and attention-deficit/ hyperactivity disorder. J Altern Complement Med. 2013;19(9):746-50.

69. D'Alò GL, De Crescenzo F, Minozzi S, Morgano GP, Mitrova Z, Scattoni ML, et al. Equity, acceptability and feasibility of using polyunsaturated fatty acids in children and adolescents with autism spectrum disorder: a rapid systematic review. Submitted to Health Qual Life Outcomes.

\section{Publisher's Note}

Springer Nature remains neutral with regard to jurisdictional claims in published maps and institutional affiliations.

Ready to submit your research? Choose BMC and benefit from:

- fast, convenient online submission

- thorough peer review by experienced researchers in your field

- rapid publication on acceptance

- support for research data, including large and complex data types

- gold Open Access which fosters wider collaboration and increased citations

- maximum visibility for your research: over $100 \mathrm{M}$ website views per year

At $\mathrm{BMC}$, research is always in progress.

Learn more biomedcentral.com/submissions 